\title{
ROLE OF MEDICAL REHABILITATION IN CAUDA EQUINA SYNDROME WITH FLACCID PARAPARESIS AFTER SPINAL MENINGITIS. A CASE REPORT
}

\author{
Gabriela Dogaru'12, Ioana Stănescu' ${ }^{12}$, Ioana Pop ${ }^{1}$ \\ 1. Clinical Rehabilitation Hospital Cluj-Napoca \\ 2. "Iuliu Hatieganu" UMPh Cluj-Napoca
}

\begin{abstract}
Patient L.I., aged 47 years, with multiple hospitalizations in November 2013 for multiple neurological and infectious diseases. In October 2011, surgery was performed for vicious posttraumatic right acetabular callus, confirmed by computed tomography. Subsequently, in November 2013, the patient had lumbar pain radiating to the lower limbs, functional impotence, sphincter incontinence, septic state with positive hemocultures for Staphylococcus aureus, with multiple paravertebral abscesses involving the left iliopsoas muscle, confirmed by contrast magnetic resonance imaging of the dorsolumbar spine, operated phlegmon of the left leg, which were interpreted as diffuse secondary spinal meningitis, complicated by a cauda equina syndrome, for which adequate antibiotic treatment was administered at the Clinic of Infectious Diseases Cluj-Napoca. The patient also presented two episodes of Clostridium difficile acute enterocolitis, with two fecal transplant sessions. In May 2014, the patient was admitted to the Rehabilitation Hospital Cluj-Napoca for motor deficit of the lower limbs, walking disorders, micturition disorders, sexual dynamic disorders, pain in the lumbar spine radiating to the lower limbs, sudden onset sensitivity disorders at D10 level in a febrile context in November 2013, interpreted based on lumbar MRI as spinal meningitis secondary to dorsal and lumbar paravertebral abscesses. During the course of hospitalization, the patient received a complex medical rehabilitation treatment consisting of kinetotherapy (posturations, passive mobilizations, active mobilizations, transfers, proprioceptive neuromuscular facilitation techniques, walking rehabilitation, respiratory gymnastics, rehabilitation of sensitivity disorders), occupational therapy, massage, medium frequency currents for the rehabilitation of micturition disorders, with good results particularly in walking and urinary incontinence rehabilitation. The aim of the presentation of this case is to emphasize the fact that rehabilitation programs using physicalkinetic therapeutic methods are the only way to limit functional deficit, playing an important role in the long-term management and care of the patient. The particularity of this case consists of the fact that the patient is young, without a known personal pathological history, and symptoms occurred two years after orthopedic surgery, with sudden motor deficit in the lower limbs. The regular follow-up of the patient and his inclusion in complex medical rehabilitation programs in order to limit the functional deficit, increase the autonomy and improve the quality of life of this patient are important.
\end{abstract}

Key words: paraparesis, rehabilitation, spinal meningitis 


\section{Introduction. Aim}

Infections involving the spinal canal include epidural abscesses (infection in the epidural space), meningitis (infection of the meninges), subdural abscesses (infections of the subdural space), and intramedullary abscesses (infections within the spinal cord).

Spinal epidural abscesses occur more frequently in the larger posterior epidural space (1). A spinal epidural abscess threatens the spinal cord or cauda equina by compression and also by vascular compromise. The more clinically significant effects of the epidural abscess may be from involvement of the vascular supply to the spinal cord and subsequent infarction rather than direct compression.

If untreated, an expanding suppurative infection in the spinal epidural space impinges on the spinal cord, producing sensory symptoms and signs, motor dysfunction, and, ultimately, paralysis and death. Intervention early in the course of the disease improves the outcome.Hematogenous spread with seeding of the epidural space is the suspected source of infection in most children and is thought to occur in many adults as well. Reported sources of infection are numerous and include bacterial endocarditis, infected indwelling catheters, urinary tract infection, peritoneal and retroperitoneal infections, and others. Direct extension of infection from vertebral osteomyelitis occurs in adults and rarely in children. Epidural catheters and injections may lead to direct inoculation of the epidural space. The source of infection is not identified in many patients. Staphylococcus aureus is the most commonly reported pathogen, though many other bacteria have been implicated, including Staphylococcus and Pseudomonas species, Escherichia coli, Brucella, and Mycobacterium tuberculosis.

Methicillin-resistant Staphylococcus aureus (MRSA) is increasingly reported particularly in patients with a history of MRSA abscesses, spinal surgery, or implanted devices. Immunosuppressed patients may have infections from unusual bacterial or fungal organisms.Cases are frequently reported in patients with alcoholism, diabetes mellitus and conditions involving chronic immunosuppression. Clinical presentation may be quite variable. The clinical triad of fever, back pain, and neurologic deficit is the classical presentation, but it could be missing in many patients (2), (3). A 4-phase sequential evolution has been described, with (a) localized spinal pain, (b) radicular pain and paresthesias, (c) muscular weakness, sensory loss, and sphincter dysfunction, and finally (d) paralysis. Abscesses from hematogenous spread tend to progress rapidly, while abscesses from osteomyelitis or discitis may evolve over weeks or months with slow progression of symptoms.

Symptoms may include the following (1), (4): fever, present in only about one third of patients, localized back pain in most patients, often the first symptom, radiculopathy with radiating or lancinating pain, including chest or abdominal pain, spinal cord syndromes, cauda equina syndrome in lumbar abcesses.Diagnostic workup include immediate imaging of the spine and spinal cord by MRI when the spinal abscess is clinically suspected. Lumbar puncture (LP) is relatively contraindicated in these situations. However, LP may be essential to exclude meningitis from the differential diagnosis. Cerebrospinal fluid (CSF) may show inflammatory cells, often a mixture of polymorphonuclear and mononuclear cells. Cell counts usually are increased, ranging from 10-1000 leukocytes/ $\mu \mathrm{L}$; CSF protein usually is elevated above $100 \mathrm{mg} / \mathrm{dL}$. Treatment most often consists of both medical-and surgical therapy (5), (6). Empiric antibiotic coverage should include antistaphylococcal antibiotics. If the infection follows a neurosurgical procedure, an antistaphylococcal penicillin, a thirdgeneration cephalosporin, and an aminoglycoside are prescribed in combination. Culture results guide definitive therapy. Deterioration of clinical and 
functional status while undergoing antibiotic therapy alone has been observed and may dictate surgical treatment (7). Emergency early decompression, irrigation, and debridement $\mathrm{s}$ with drainage of the abscess are the manstay of the surgical treatment.

The aim of the presentation of this case is to emphasize the fact that rehabilitation programs using physical-kinetic therapeutic methods are the only way to limit functional deficit, playing an important role in the longterm management and care of the patient.

\section{Material and method}

Patient L.I., aged 47 years, with multiple hospitalizations in November 2013 for multiple neurological and infectious diseases. In October 2011, surgery was performed for vicious posttraumatic right acetabular callus, confirmed by computed tomography. Subsequently, in November 2013, the patient had lumbar pain radiating to the lower limbs, functional impotence, sphincter incontinence, septic state with positive hemocultures for Staphylococcus aureus, with multiple paravertebral abscesses involving the left iliopsoas muscle, confirmed by contrast magnetic resonance imaging of the dorsolumbar spine, operated phlegmon of the left leg, which were interpreted as diffuse secondary spinal meningitis, complicated by a cauda equina syndrome, for which adequate antibiotic treatment was administered at the Clinic of Infectious Diseases Cluj-Napoca. The patient also presented two episodes of Clostridium difficile acute enterocolitis, with two fecal transplant sessions. In May 2014, the patient was admitted to the Rehabilitation Hospital Cluj-Napoca for motor deficit of the lower limbs, paresthesia of the lower limbs, walking disorders, micturition disorders, sexual dynamic disorders, pain in the lumbar spine radiating to the lower limbs, sudden onset sensitivity disorders at D10 level in a febrile context in November 2013, interpreted based on lumbar MRI as spinal meningitis secondary to dorsal and lumbar paravertebral abscesses.

On the general objective examination, no changes were found. $\mathrm{BP}=120 / 70 \mathrm{mmHg}$, $\mathrm{VR}=72$ beats/min, normally shaped abdomen, without sensitivity to palpation.

The objective examination of the locomotor system revealed pain on palpation in the lumbar spine, with the limitation of mobility, with a Schober test of $12 / 15 \mathrm{~cm}$, crepitations on knee mobilization. The objective neurological examination evidenced walking with a wide weight bearing base, possibly without support, muscle testing showed left plantar dorsiflexion $4 / 5$, left plantar flexion $4 / 5$, normal osteotendinous reflexes in the upper limbs, no Achilles reflexes, diminished tactile sensitivity in the right L3-L4 and left L1-L2 areas, partial urinary incontinence, sexual dynamic disorders.

Paraclinical investigations performed: biological tests within normal limits. The Xray of the lumbosacral spine evidenced mild lumbar scoliosis, spondylotic lumbar changes.

Drug treatment administered: NSAIDs, vitamins, eubiotic therapy. The patient was evaluated clinically and functionally at the beginning of rehabilitation treatment, as well as after two weeks of treatment. Pain using the visual analogue scale (VAS), joint mobility by articular check-up, muscle strength through muscle testing, and transfers, walking, micturition disorders were evaluated. During the course of hospitalization, the patient received a complex medical rehabilitation treatment consisting of kinetotherapy (posturations, passive mobilizations, active mobilizations, transfers, proprioceptive neuromuscular facilitation techniques, walking rehabilitation, respiratory gymnastics, rehabilitation of sensitivity disorders), occupational therapy, massage, medium frequency currents for the rehabilitation of micturition disorders. 


\section{Results}

After two weeks of drug treatment and rehabilitation, pain intensity in the lumbar spine was reduced (VAS before treatment 8 , after treatment 4), lumbar mobility increased, $14 / 15 \mathrm{~cm}$, with better results in the rehabilitation of walking and urinary incontinence. The study revealed the benefits of physical-kinetic therapy in the maintenance and increase of joint mobility, relief of pain, improvement of muscle strength, respiratory function, micturition disorders.

\section{Conclusions}

Rehabilitation programs using physicalkinetic therapeutic methods: massage, kinetotherapy, electrotherapy, occupational therapy represent the only way to limit functional deficit, playing an important role in the long-term management and care of the patients. The particularity of this case consists of the fact that the patient is young, without a known personal pathological history, and symptoms occurred two years after orthopedic surgery, with sudden motor deficit in the lower limbs. The regular follow-up of the patient and his inclusion in complex medical rehabilitation programs in order to limit the functional deficit, increase the autonomy and improve the quality of life of this patient are important.

\section{Bibliography}

1. Darouiche RO. Spinal epidural abscess. $N$ Engl $J$ Med. Nov 9 2006;355(19):2012-20

2. Joshi SM, Hatfield RH, Martin J, Taylor W. Spinal epidural abscess: a diagnostic challenge. $\mathrm{Br} J$ Neurosurg. Apr 2003;17(2):160-3.

3. Davis DP, Wold RM, Patel RJ, et al. The clinical presentation and impact of diagnostic delays on emergency department patients with spinal epidural abscess. $J$ Emerg Med. Apr 2004;26(3):285-91

4. Dugas AF, Lucas JM, Edlow JA. Diagnosis of spinal cord compression in nontrauma patients in the emergency department. Acad Emerg Med. Jul 2011;18(7):719-25

5. Siddiq F, Chowfin A, Tight R, et al. Medical vs surgical management of spinal epidural abscess. Arch Intern Med. Dec 1327 2004;164(22):2409-12.

6. Pradilla G, Ardila GP, Hsu W, Rigamonti D. Epidural abscesses of the CNS. Lancet Neurol. Mar 2009;8(3):292300 .

7. Patel AR, Alton TB, Bransford RJ, Lee MJ, Bellabarba CB, Chapman JR. Spinal epidural abscesses: risk factors, medical versus surgical management, a retrospective review of 128 cases. Spine J. Feb 1 2014;14(2):326-30 